\title{
Myo-Inositol Safety in Pregnancy: From Preimplantation Development to Newborn Animals
}

\author{
Nilay Kuşcu, ${ }^{1}$ Mariano Bizzarri, ${ }^{2}$ and Arturo Bevilacqua ${ }^{1,3}$ \\ ${ }^{1}$ Department of Psychology, Section of Neuroscience, Sapienza University of Rome, Via dei Marsi 78, 00185 Rome, Italy \\ ${ }^{2}$ Department of Experimental Medicine, Sapienza University of Rome, Viale Regina Elena 324, 00161 Rome, Italy \\ ${ }^{3}$ Research Center in Neurobiology Daniel Bovet (CRiN), 00185 Rome, Italy
}

Correspondence should be addressed to Arturo Bevilacqua; arturo.bevilacqua@uniromal.it

Received 22 June 2016; Accepted 15 August 2016

Academic Editor: Nikos Prapas

Copyright (C) 2016 Nilay Kușcu et al. This is an open access article distributed under the Creative Commons Attribution License, which permits unrestricted use, distribution, and reproduction in any medium, provided the original work is properly cited.

\begin{abstract}
Myo-inositol (myo-Ins) has a physiological role in mammalian gametogenesis and embryonic development and a positive clinical impact on human medically assisted reproduction. We have previously shown that mouse embryo exposure to myo-Ins through preimplantation development in vitro increases proliferation activity and blastocyst production, representing an improvement in culture conditions. We have herein investigated biochemical mechanisms elicited by myo-Ins in preimplantation embryos and evaluated myo-Ins effects on postimplantation/postnatal development. To this end naturally fertilized embryos were cultured in vitro to blastocyst in the presence or absence of myo-Ins and analyzed for activation of the PKB/Akt pathway, known to modulate proliferation/survival cellular processes. In parallel, blastocyst-stage embryos were transferred into pseudopregnant females and allowed to develop to term and until weaning. Results obtained provide evidence that myo-Ins induces cellular pathways involving Akt and show that (a) exposure of preimplantation embryos to myo-Ins increases the number of blastocysts available for uterine transfer and of delivered animals and (b) the developmental patterns of mice obtained from embryos cultured in the presence or absence of myo-Ins, up to three weeks of age, overlap. These data further identify myo-Ins as a possibly important supplement for human preimplantation embryo culture in assisted reproduction technology.
\end{abstract}

\section{Myo-Inositol}

Myo-inositol (myo-Ins) is the major component of a family of nine hexahydroxycyclohexane inositol (Ins) stereoisomers broadly distributed in eukaryotic tissues and cells, where they are involved in basic biological functions [1].

Ins is incorporated into cell membrane phosphoinositides or phosphatidyl-glycerides as phosphatidyl-inositol (PI), which is phosphorylated by a set of specific phosphoinositide-3-kinases (PI3K) to phosphatidyl-inositol phosphates. Phosphatidyl-inositol trisphosphate (PIP3) can be further metabolized by phospholipase C (PLC) to inositol trisphosphate (Ins-1,4,5P3, InsP3), which acts as second messenger for various hormones such as FSH, TSH, and insulin $[2,3]$. Inositol is the precursor of other membrane components and glycosyl-phosphatidyl-inositol (GPI) protein-anchor molecules (reviewed by [4]), signaling molecules (reviewed by $[5,6])$, chromatin-remodeling, and transcription-modulating molecules [7]. It finally acts as a hyperosmotic stress protectant [8].

\section{Myo-Inositol in \\ Reproduction, Gametogenesis, and Embryonic Development}

Increasing evidence shows that myo-Ins has a physiological role in mammalian gametogenesis and embryonic development and a positive clinical impact on human medically assisted reproduction.

Together with its isomer D-chiro-inositol (D-chiro-Ins), myo-Ins acts as second messenger of insulin [9] and has been widely studied for its involvement in metabolic syndrome (reviewed by [10]) as well as for the treatment of polycystic 
ovary syndrome (PCOS) (reviewed by [11, 12]), one of the most common female endocrine disorders [13] strictly associated with insulin resistance [14]. Recent reports strongly suggest that both Ins isomers can be positively associated in the management of PCOS patients enrolled in assisted reproduction procedures [15], with significant encouraging clinical outcomes [16].

While D-chiro-Ins does not appear to have a clear role in the ovary or in the testis, myo-Ins affects gametogenetic and embryogenetic processes at several levels, having a protective, positive role in reproduction and development (reviewed by [17]).

Myo-Ins concentration in the reproductive tracts of male mammals is higher than in blood serum being produced by FSH-responsive Sertoli cells [18, 19]. Studies performed on pathological sperm samples have shown that myo-Ins is crucial in spermatogenesis by positively affecting sperm cell motility [20] and mitochondrial membrane potential [21], an apoptotic marker directly associated with cell viability. Since these parameters are predictors of good embryos quality, the use of myo-Ins in the andrology laboratory is now employed to improve the recovery of sperm cells after swim-up in in vitro fertilization (IVF) cycles [20].

As for female gametogenesis, myo-Ins is actively concentrated in mammalian follicular and tubal fluid, being higher than in blood serum [22]. Respective values of myoIns concentration in human follicular fluid and serum are 30to- $40 \mu \mathrm{M}$ [23] and 10-to- $20 \mu \mathrm{M}$ [24]. In addition, a positive correlation exists between follicular myo-Ins content and oocyte quality as well as pregnancy outcome [23].

At the ovarian level, Myo-Ins has different functions in both follicle cells and oocytes. However, although it is known that myo-Ins metabolism is severely deregulated in follicle cells of PCOS patients [25], effects of this molecule on somatic ovarian cells still need to be evaluated. We have hypothesized that in theca and granulosa cells myo-Ins may sustain steroidogenic activity during the ovarian cycle by modulating the dynamics of cytoskeletal structures [26]. Research on this issue is ongoing in our laboratories.

Myo-Ins is transported into mammalian cells, including growing and fully-grown oocytes and early preimplantation embryo blastomeres, by a sodium cotransporter [27] and a sodium independent transporter [28]. In oocytes, activity of myo-Ins-derived InsP3 on the modulation of intracellular $\mathrm{Ca}^{2+}$ concentration in response to the action of the hormones LH and FSH $[29,30]$ is well known. It acts via cell-specific receptors (InsP3-R1) [31] and appears to play a key role in meiotic maturation [32].

Chiu et al. [33] cultured preovulatory oocytes from outbred mice in minimal essential medium supplemented with myo-Ins and observed increased rates of maturation, IVF, and development to the two-cell stage with respect to oocytes cultured in medium alone, suggesting that higher availability of myo-inositol increases both meiotic and activation efficiencies of the oocyte. After transfer to foster mothers, the implantation rate and postimplantation viability of these embryos were also increased in the myo-Ins treated groups [33].
During mammalian preimplantation development, activity of myo-Ins transporters ensures a robust uptake, which progressively increases from the one-cell to the blastocyst stage $[34,35]$, suggesting a parallel increase in cellular requirement of the molecule. It has been shown that, in preimplantation embryo blastomeres, myo-Ins is rapidly incorporated into phosphoinositides [34], leading to raised intracellular InsP3 levels. This led several groups, including ours, to investigate whether myo-Ins could also have a positive action during preimplantation development in both the laboratory mouse and farming species.

Myo-Ins supplementation of culture media was found to improve rabbit and bovine blastocyst formation and expansion $[35,36]$. Culture of rabbit embryos at the morula stage in medium containing myo-Ins at the optimal concentration of $75 \mu \mathrm{M}$ resulted in blastocysts expansion with a fourfold increase in diameter when compared to that provided by standard culture conditions [35]. Similar observations were obtained on bovine zygotes matured and fertilized in vitro [36], after culture in synthetic oviduct fluid medium [37] in the presence or absence of $2.77 \mathrm{mM}$ myo-Ins. As a result, blastocyst rate was higher among embryos developed in the presence of myo-Ins [36]. To evaluate postimplantation effects of this treatment, ten blastocysts grown in the presence of myo-Ins were transferred to foster cows and developed to term, producing five healthy animals [36].

With a clear interest in human assisted reproduction technology (ART), positive data on myo-Ins in mammalian preimplantation development prompted us to hypothesize that inclusion of myo-Ins in embryo culture media would result in increased numbers of high quality embryos produced by IVF/intracytoplasmic sperm injection (ICSI). We tested this hypothesis in a previous work [38], using the mouse embryo model $[39,40]$, by investigating the effects of myo-Ins supplementation of sequential human embryo culture media, starting 30 minutes after fertilization (p.f.) and for the whole length of preimplantation development (day 4 p.f.). In that study, we cultured embryos obtained by ICSI from gametes of inbred C57BL/6N mice, in which ICSI results in suboptimal rates of oocyte survival and blastocyst development, when compared to hybrid mice [41]. After fertilization in unmodified fertilization medium, embryos were cultured in cleavage medium in the presence or absence of $10 \mathrm{mM}$ myo-Ins (myo-Ins+ and myo-Ins-, resp.) and monitored daily for developmental progression. At all-time intervals monitored, (p.f.), myo-Ins+ embryos displayed a faster cleavage rate, with a higher percentage of embryos at the most advanced stage. In these embryos, early differentiative events such as compaction and blastulation occurred at the proper developmental stage, excluding apparent toxic effects of myo-Ins. Although results scored on day 4 have already been published [38], those recorded at intermediate times have not been presented elsewhere and are reported in Figures 1 and 2, together with embryo morphology observed on day 4.

Embryos cultured in the presence of myo-Ins were developmentally advanced with respect to control embryos, being mostly represented by expanded blastocysts with a higher number of blastomeres, as shown by Hoechst 33343 nuclear 


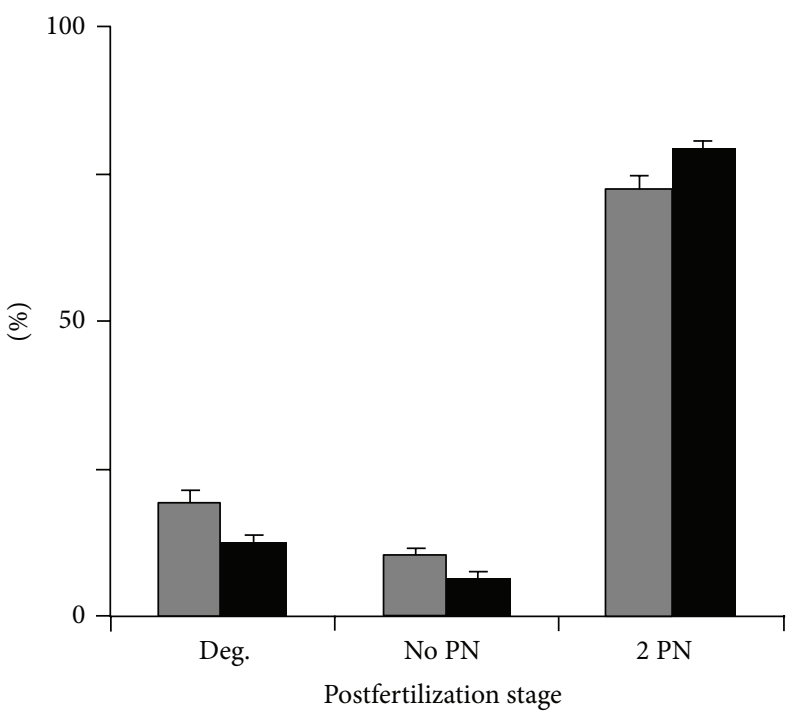

(a)

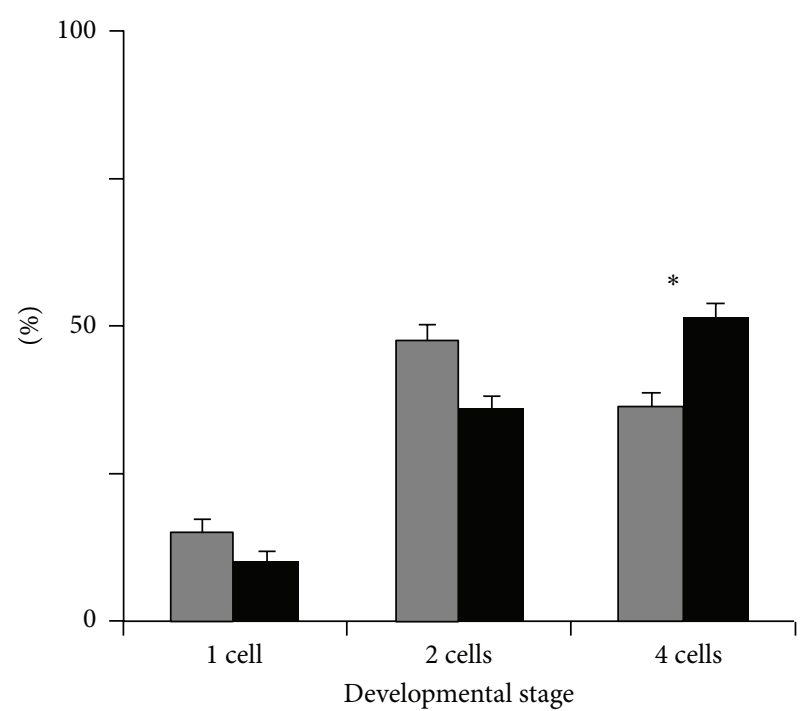

(b)

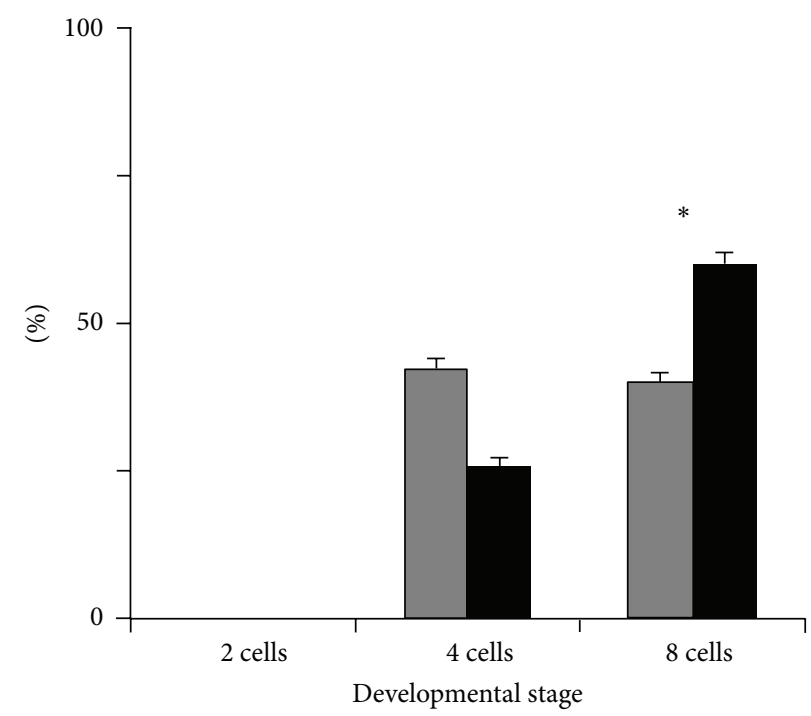

(c)

FIGURE 1: Effect of myo-inositol medium supplementation on pronuclear formation and embryo development during the first two days of in vitro culture. Completion of early developmental steps by zygotes cultured in the absence (grey bars) or the presence (solid bars) of $10 \mathrm{mM}$ myo-inositol. Zygotes were scored 6 hours (a) and embryos at 24-26 hours (b) and 48-50 hours (c) p.f. Bars represent the mean \pm SEM of 7 independent experiments of the fraction of embryos at each indicated stage. (a) N, myo-Ins- embryos (grey bars), 121; myo-Ins+ embryos (solid bars), 123. ((b), (c)) N, myo-Ins- embryos (grey bars), 97; myo-Ins+ embryos (solid bars), 106. Asterisks indicate difference between treatments calculated by $\chi^{2}$ test with Yates correction for continuity: (b) $P<0.05$; (c) $P<0.01$.

staining [38]. We concluded that myo-Ins supplementation represents an improvement of culture conditions reducing the developmental gap typically observed between embryos obtained and cultured in vitro and those developed in vivo, further supporting its possible use for human embryo preimplantation culture.

One of the issues left uncovered by these experiments concerns the nature of biochemical pathways induced by exposure to myo-Ins in preimplantation of embryo blastomeres. Existing information suggests that possible pathway(s) elicited by myo-Ins in mouse embryo blastomeres and responsible for a reduced length of cell cycles and a more efficient proliferation activity are initiated via rapid incorporation into phosphoinositides [34], namely, PIP3, enzymatically fostered by activity of PI3K. In turn, PIP3 is converted by PLC into diacylglycerol and InsP3, which, by mobilizing $\mathrm{Ca}^{2+}$ stores, has already been recognized to speed up several cellular processes, including proliferation $[42,43]$. PI3K is also responsible for the activation of the $\mathrm{PKB} /$ Akt pathway [44], which is known to promote proliferation of mouse embryo blastomeres [45]. As myo-Ins has been shown to increase Akt phosphorylation and hence its activity in mouse skeletal muscle cells [46], we hypothesized that a critical step in enhancing embryo preimplantation 


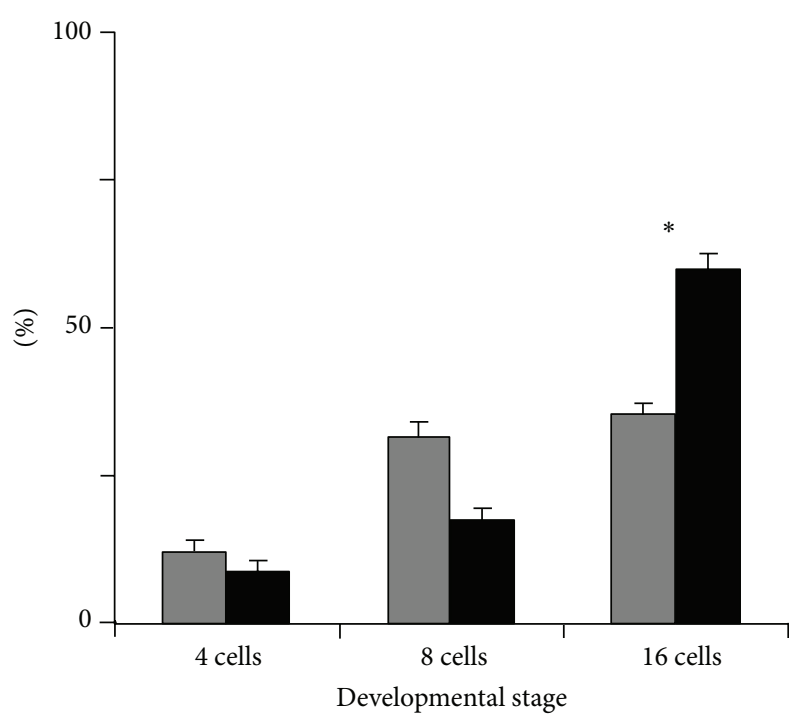

(a)

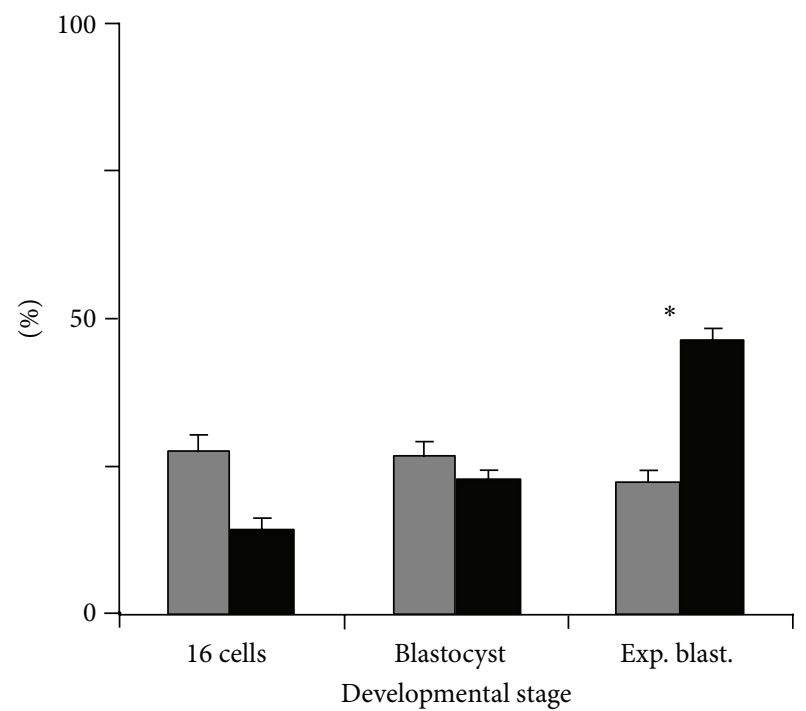

(b)
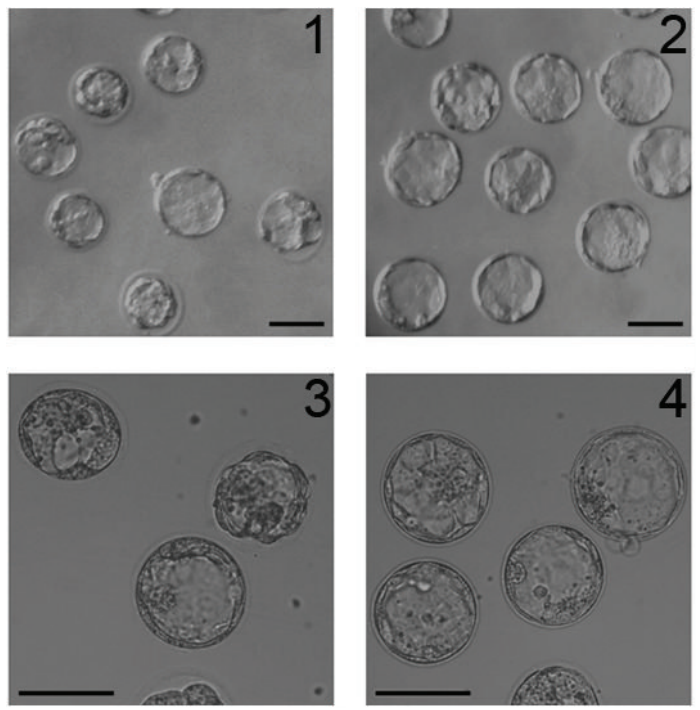

(c)

FIGURE 2: Effect of myo-inositol medium supplementation on embryo development during the third and fourth days of in vitro culture. Completion of mid-to-late preimplantation development by embryos cultured in the absence (grey bars) or the presence (solid bars) of $10 \mathrm{mM}$ myo-inositol. Embryos were scored 72-74 hours (a) and 96-98 hours (b) p.f. Bars represent the mean \pm SEM of 7 independent experiments of the fraction of embryos at each indicated stage. ((a), (b)) N, myo-Ins- embryos (grey bars), 97; myo-Ins+ embryos (solid bars), 106. Exp. blast.: expanded blastocyst. Asterisks indicate difference between treatments calculated by $\chi^{2}$ test with Yates correction for continuity: (a) $P<0.05$; (b) $P<0.05$. (c) Representative images of expanded and nonexpanded blastocysts observed at day 4 among myo-Ins- $(1,3)$ and myo-Ins+ $(2,4)$ embryos; magnification: 40x $(1,2) ; 80 \mathrm{x}(3,4)$. Black bars, $100 \mu \mathrm{m}$. Panel (b) is modified from [38].

development may be represented by Akt activation induced by myo-Ins supplementation [47]. We here provide the first evidence, obtained by immunofluorescence analysis, that myo-Ins supplementation of culture media increases serine phosphorylation of $\mathrm{PKB} / \mathrm{Akt}$ in mouse preimplantation embryos.

A second open issue in current research concerns the safety of preimplantation embryo exposure to myo-Ins for postimplantation and postnatal development.

Besides the reported observations on bovine embryos [36], it could be argued that preimplantation embryos, including human embryos, were once routinely cultured in undefined media supplemented with commercial BSA preparations or batches of bovine or human sera, which contain variable amounts of myo-inositol, without reporting any apparent negative effect.

In addition, myo-Ins appears important for normal development (reviewed by [17]). Its concentration in foetal human serum is severalfold higher than in adults, lowering only around birth [48], and its administration during pregnancy has positive effects on pathological conditions in both humans $[49,50]$ and rodent species [51-54]. 
All data hitherto reported suggest that preimplantation embryo exposure to myo-Ins would have no detrimental effects on further development. To obtain more direct information on this issue, we transferred blastocysts produced in vitro in the presence of myo-Ins into recipient foster mothers and allowed their development to term. By this approach, we obtained healthy offspring that appeared normal in the sex ratio and, at least until weaning, somatometrically. These experiments provide additional data on myo-Ins effects on mammalian preimplantation embryos and strongly suggest that it can be considered safe for embryo development to term.

\section{Materials and Methods}

3.1. Animals. Animals (Charles River Italia, Calco, VA, Italy) were housed in a temperature-controlled facility $\left(22 \pm 1^{\circ} \mathrm{C}\right)$ on a $12 / 12 \mathrm{~h} \mathrm{light/dark}$ cycle, inside standard cages with unlimited access to food and water. Forty-to-60-day-old C57BL/6N mice were used as donors of oocytes and male studs. Forty-to-60-day-old CD/1 mice were used as foster mothers and vasectomized male studs. All experimental procedures were conducted in accordance with the official European guidelines for the care and use of laboratory animals (86/609/EEC). Experimental protocols and related procedures were approved by the Italian Ministry of Public Health.

3.2. Culture Media. In order to model human preimplantation embryo culture, all procedures were performed in commercial Quinn's Advantage media and supplements (BioCare Europe, Napoli, Italia), which do not contain myo-Ins, as described [38].

3.3. Animal Treatment and Zygote Collection. Female mice were hormonally induced by intraperitoneal injections of $5 \mathrm{IU}$ pregnant mare serum gonadotropin (NHPP, Torrence CA, USA) and, $46-48 \mathrm{~h}$ later, of $5 \mathrm{IU}$ human chorionic gonadotropin (Corulon, Intervet Italia, Aprilia, LT, Italy). After mating with male mice $\mathrm{ON}$, females carrying a vaginal plug were sacrificed by cervical dislocation and cumulusoocyte complexes were collected from the oviducts, freed from cumulus cells by treatment with $0.1 \%$ hyaluronidase in Hepes buffered Quinn's Protein Plus Advantage HTFMedium containing 5\% human serum albumin, rinsed in HTF-Medium, and incubated at $37^{\circ} \mathrm{C}$ in Quinn's Protein Plus Cleavage Medium (C-Medium) under a humidified atmosphere of $5 \% \mathrm{CO}_{2}$ in air.

3.4. Embryo Culture. One-cell embryos were positively scored by the presence of two pronuclei and then divided into two groups. One group was further cultured in $14 \mu \mathrm{L} / \mathrm{mL}$ myo-inositol (10 mM, Andrositol ${ }^{\circledast}$ LAB Lo.Li. Pharma, Italy) in C-Medium and the other one in $14 \mu \mathrm{L} / \mathrm{mL}$ phosphate buffered saline in C-Medium. Embryo culture was performed in $500 \mu \mathrm{L}$ medium inside Falcon 4 well IVF plates without medium replacement, as described [38]. Developing embryos were scored daily for morphology and progression through cleavage stages.
3.5. Detection of Phosphorylated PKB/Akt. Preimplantation embryos at the morula or blastocyst stage were washed in $0.1 \mathrm{M}$ phosphate buffered saline, $\mathrm{pH} 7.4$ (PBS), containing $0.3 \%$ bovine serum albumin (BSA) (PBS-BSA), and were then fixed in $4 \%$ paraformaldehyde in PBS for $30 \mathrm{~min}$ at room temperature (RT). After three washes in PBS-BSA, embryos were permeabilized in PBS containing 0.25\% Tween-20 (Sigma; St. Louis, MO) for $15 \mathrm{~min}$ at RT and washed three times in PBSBSA. Permeabilized embryos were processed for immunostaining by an overnight incubation at $4^{\circ} \mathrm{C}$ in the presence of the primary antibody, rabbit monoclonal anti-phosphoAkt (Ser473, \#4080), anti-phospho-Akt (Thr308, \#4056) or anti-Akt (total, \#9272) antibody (Cell Signaling Technology, Beverly, MA, USA) (1:75), followed by washing in PBS-BSA and $1 \mathrm{hr}$ final incubation with the secondary, and Alexa Flour488 conjugated secondary antibodies (Invitrogen), diluted 1:200 in PBS-BSA for $1 \mathrm{~h}$ at RT. After washing with PBSBSA, blastomere nuclei were counterstained with $1 \mathrm{mg} / \mathrm{mL}$ Hoechst 33343 for 10 min at RT. Finally, embryos were washed three times for 10 min each in PBS-BSA and mounted in PBS and glycerol $(1: 1 \mathrm{v} / \mathrm{v})$ using coverslips. Fluorescent signals were detected using a Zeiss AxioPlan fluorescence microscope (Carl Zeiss, Oberkochen, Germany) at 400x magnification. For semiquantitative analysis of fluorescence, embryos at various developmental stages were immunostained after pooling in the same drops. Fluorescence emission was collected under similar excitation conditions and then quantitatively analyzed by using the ImageJ software (ImageJ $1.47 \mathrm{v}$, Wayne Rasband, National Institutes of Health, USA. http://imagej.nih.gov/ij/).

3.6. Embryo Transfer and Development to Term. In ten replicate experiments, embryos developed to the blastocyst stage after 4 days of culture in myo-Ins+ C-Medium or myo-InsC-Medium were transferred to the uteri of pseudopregnant foster mothers mated 2.5 days earlier with vasectomized males and carried to term [55]. On the day of delivery, newborn animals were weighed, checked for gross abnormalities, and left to be nursed by their moms until weaning. Preweaning morphological analyses included body growth at one week, fur appearance, and eye opening. Mice were finally weighed and sexed at weaning (three weeks of age).

3.7. Chemicals. Where not stated otherwise, chemicals were purchased from Sigma-Aldrich Co. (St. Louis, MO, USA).

3.8. Data Analysis. Data were analyzed by using Student's $t$ test or $\chi^{2}$ tests with Yates continuity correction. Statistical analyses were performed using R: A language and environment for statistical computing ( $\mathrm{R}$ development core team, $\mathrm{R}$ foundation for statistical computing, ISBN 3-900051-07-0, 2008, Vienna, Austria. https://www.r-project.org/).

\section{Results}

4.1. Analysis of Myo-Ins-Dependent Akt Phosphorylation. We have determined presence and phosphorylation of Akt in late preimplantation stage embryos cultured in the presence 




(a)

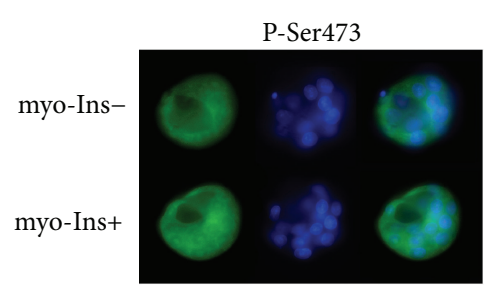

(c)

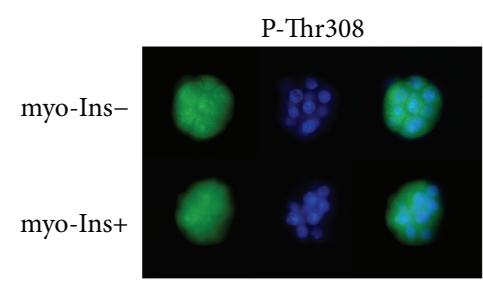

(b)

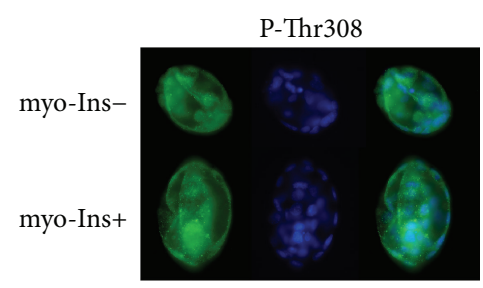

(d)

FiguRE 3: Localization of phosphorylated Akt in preimplantation embryos at the morula and blastocyst stage, after continuous culture in the presence or absence of myo-Ins. ((a), (b)) morula stage embryos; ((c), (d)) blastocyst stage embryos. In each panel, from left to right: FITC, Hoechst 33343, merge. The same pattern was consistently observed in all embryos analyzed; panels show representative embryos. Statistical analysis on these embryos, reported in the text, was performed on at least three embryos at the morula and blastocyst stages in three independent experiments.

TABLE 1: Effect of myo-Ins on Akt phosphorylation of late preimplantation mouse embryos.

\begin{tabular}{cccc}
\hline \multicolumn{2}{c}{ Morulae } & \multicolumn{2}{c}{ Blastocysts } \\
Ser473 & Thr308 & Ser473 & Thr308 \\
\hline Myo-Ins+ $65.41 \pm 5.01$ & $47.77 \pm 4.37$ & $49.33 \pm 6.16$ & $32.15 \pm 1.56$ \\
Myo-Ins- 59.80 \pm 2.55 & $45.87 \pm 2.23$ & $38.32 \pm 2.54^{\mathrm{a}}$ & $33.09 \pm 2.03$ \\
\hline
\end{tabular}

Data represent arbitrary fluorescence units (mean \pm SD) of embryos at the stages indicated, pooled from three replicate experiments.

${ }^{\mathrm{a}} P=0.02$, calculated by Student's $t$-test.

or absence of myo-Ins by immunofluorescence analysis. This approach revealed the presence of serine 473- and threonine 308-phosphorylated Akt in both morula and blastocyst embryos (Figure 3). Phosphorylated Akt was localized prominently in blastomere cytosols but a limited nuclear localization was also observed. The relative content of phosphorylated Akt at the same stages was also measured by quantification of immunofluorescence data (Table 1). The level of serine 473-phosphorylated Akt did not appear to be modified in embryos cultured in the presence of myo-Ins at the morula stage $(t 4=1.75, P=0.15)$ (Figure 3(a)) but it was increased at the blastocyst stage $(t 4=3.61, P=0.02)$ (Figure 3(c)). On the contrary, the level of threonine 308phosphorylated Akt did not vary significantly depending on the presence or absence of myo-Ins (morulae, $t 4=0.670$, $P=0.54$, Figure 3(b); blastocysts, $t 4=0.640, P=$ 0.56 , Figure $3(d))$. Amounts of total Akt did not vary under different conditions (not shown).

4.2. Effects of Preimplantation Embryo Exposure to Myo-Ins on Development to Term. In ten replicate experiments, all embryos that had developed in vitro to the expanded or nonexpanded blastocyst stage in the presence or absence
TABLE 2: Mouse development after IVF and exposure of preimplantation embryos to myo-Ins.

\begin{tabular}{lccc}
\hline & $\begin{array}{c}\text { Number of } \\
\text { fertilized } \\
\text { oocytes }\end{array}$ & $\begin{array}{c}\text { Number of } \\
\text { transferred } \\
\text { blastocysts (\%) }\end{array}$ & $\begin{array}{c}\text { Number of } \\
\text { delivered } \\
\text { animals (\%) }\end{array}$ \\
\hline Myo-Ins+ & 154 & $105(68.2)$ & $59(38.3)$ \\
Myo-Ins- & 147 & $76(51.7)^{\mathrm{a}}$ & $33(22.5)^{\mathrm{a}}$ \\
\hline
\end{tabular}

Data were pooled from ten replicate experiments.

Superscripts indicate differences between myo-Ins+ and myo-Ins- embryos $(P<0.05)$ calculated by $\chi^{2}$ test; differences are referred to fertilized oocytes.

of myo-Ins were transferred to foster mothers and allowed to develop through birth and until weaning. Of 105 myoIns+ and 76 myo-Ins- blastocysts transferred, 59 (56.2\%) and $33(43.4 \%)$, respectively, were delivered $\left(\chi^{2}=2.876\right.$, $P=0.09)$. Although this difference was not significant, when we compared numbers of delivered animals with numbers of fertilized oocytes cultured under the two conditions, 154 myo-Ins+ one-cell embryos and 147 myo-Ins- onecell embryos, we obtained a significant improvement in the overall efficiency of the treatment $\left(\chi^{2}=8.91, P=0.003\right)$. A similar difference was observed by comparing numbers of fertilized oocytes with numbers of transferred embryos $\left(\chi^{2}=8.52, P=0.003\right)$. Overall results are shown in Table 2 .

Body weights at birth were $1.42 \pm 0.01 \mathrm{~g}$ for myo-Ins+ and $1.41 \pm 0.02 \mathrm{~g}$ for myo-Ins- $(t 90=0.645, P=0.52)$. During the first week, six and four pups were found dead in the myo-Ins+ and myo-Ins- groups, respectively. Somatometric development appeared similar in mice of both groups, with appropriate acquisition of body fur and eye opening. Weights at one week of age were also similar (myo-Ins+, $2.06 \pm$ $0.16 \mathrm{~g}$, myo-Ins-, $2.08 \pm 0.19 \mathrm{~g} ; t 80=0.286, P=0.78)$. Sex distributions in the two conditions were both casual 
(myo-Ins+, 27 males, 26 females, myo-Ins-, 14 males, 15 females). Finally, weights at three weeks of age were similar for sexes and embryo culture conditions (males: myo-Ins+, $10.20 \pm 0.21 \mathrm{~g}$; myo-Ins $-, 10.29 \pm 0.28 \mathrm{~g}, t 39=0.790, P=0.43$; females: myo-Ins+, $9.90 \pm 0.19 \mathrm{~g}$; myo-Ins-, $9.75 \pm 0.40 \mathrm{~g}$, $t 39=1.214, P=0.23)$.

\section{Discussion}

Akt represents a major downstream effector of growth factors, cytokines, and adhesion receptors capable of promoting cell survival and proliferation $[56,57]$ and is regulated, among others, by PIP3, the second messenger product of PI3K, via recruitment of PDK1. PDK1 induces direct phosphorylation at threonine 308 [58] and appears to be also implicated in the negative regulation of phosphorylation at serine 473 , by direct binding to this hydrophobic site. However, PDK1 can be displaced by an appropriate signal [59, 60], including activation of integrin Like Kinase (ILK) [44], allowing Akt autophosphorylation or phosphorylation by other kinases at serine 473 [58]. This pathway makes Akt phosphorylation of serine 473 inducible by upstream signals; on the contrary, phosphorylation of threonine 308 appears to have a constitutive nature [56].

We have previously shown that, during the first two stages of embryo development, Akt phosphorylation is not prevented by inhibition of either PI3K or PDK1 and concluded that the phosphorylation state and the intracellular distribution of Akt in two-cell embryos are independent of the activity of both kinases. This suggested that Akt is inherited from the oocyte in its phosphorylated/dephosphorylated form at both serine 473 and threonine 308 [45].

We now show by quantitative immunofluorescence analysis that serine 473 phosphorylation of Akt can be increased in late preimplantation embryos by the presence of myoIns in the culture medium. It thus appears that, after the initial stages of development, new phosphorylation of Akt may occur in mid-to-late preimplantation stages depending on availability of myo-Ins. In agreement with previous observations that development of mouse preimplantation embryos requires PI3K activity from the $8 / 16$-cell stage [45], it would be reasonable to hypothesize that this finding can be ascribed to the increased production of phosphoinositides [34] and consequent increased production of PIP3 by PI3K. An increase in phosphorylation of Akt may be responsible for the faster developmental rate of embryos cultured in the presence of myo-Ins.

The lack of inducibility of threonine phosphorylation of Akt by myo-Ins is also in agreement with previous observations [56].

It is puzzling that myo-Ins exerts opposite effects on both PI3K and Akt activities when added to cancer cell cultures [61], in which it has been found to reduce PI3K levels as well as Akt activity by inhibiting its phosphorylation. To this respect, it is therefore tempting to speculate that such effect could be considered context-dependent and that some other still unknown factors are likely to participate in modulating the PI3K/Akt pathway. In order to obtain more specific information on this pathway, we are currently analyzing the effects of myo-Ins both on development and on Akt phosphorylation in preimplantation embryo in the presence of inhibitors of PI3K and PDK1 [45]. Further experiments will address the involvement on proliferative activity of preimplantation embryos blastomeres of pro- and anti-apoptotic factors of the Bcl-2 family [62].

Data here produced represent a first assessment of the effect of preimplantation embryo exposure to myo-Ins on mouse development to term.

So far, information on this issue in mammals is limited to one finding obtained on bovine embryos cultured in the presence of $2.77 \mathrm{mM}$ myo-Ins [36]. In that study no comparison was made between embryos cultured in the two conditions, but blastocysts that had developed after preimplantation exposure to myo-Ins were transferred producing healthy animals.

Present results obtained in the mouse show (a) the apparent absence of early toxic effects of myo-Ins, as suggested by normal prenatal and short-term postnatal development, and (b) a significant increase in the overall rate of live births obtained after preimplantation embryo culture in myo-Ins and subsequent transfer into foster mothers.

If the first observation was expected in light of the body of information here reported on the positive effects displayed by myo-Ins on mammalian gametogenesis and development, the second one deserves particular attention. In fact, it supports the possibility that a regular use of myo-Ins as culture supplement provides high efficiency in the production of viable preimplantation embryo in vitro both in the mouse and in farming specie with promising outcome for both scientific and economic purposes.

In addition, it strengthens the hypothesis that the use of myo-Ins would have a similar positive role in the culture of in vitro produced human embryos, with obvious medical consequences.

To this end, however, additional assessments of myo-Ins effects are necessary at least at three different levels [63]: (a) on the expression of imprinted genes during development; (b) on the acquisition of sensory/motor/behavioral functions during early development; and (c) on long-term consequences on the whole organism.

\section{Disclosure}

Nilay Kuşcu's present address is Histology and Embryology Department, Medical Faculty, Akdeniz University, Campus, 07070 Antalya, Turkey.

\section{Competing Interests}

The authors declare that there is no conflict of interests regarding the publication of this paper.

\section{Acknowledgments}

This work was financially supported by the "Ateneo" Grant C26A147EE2 to Arturo Bevilacqua and the Scientific King Saud University Research Grant no. RG \#164 to Mariano Bizzarri. 


\section{References}

[1] M. Bizzarri and G. Carlomagno, "Inositol: history of an effective therapy for Polycystic Ovary Syndrome," European Review for Medical and Pharmacological Sciences, vol. 18, no. 13, pp. 18961903, 2014.

[2] R. M. Thomas, C. A. Nechamen, J. E. Mazurkiewicz, A. UlloaAguirre, and J. A. Dias, “The adapter protein APPL1 links FSH receptor to inositol 1,4,5-trisphosphate production and is implicated in intracellular $\mathrm{Ca}^{2+}$ mobilization," Endocrinology, vol. 152, no. 4, pp. 1691-1701, 2011.

[3] H. Grasberger, J. Van Sande, A. H.-D. Mahameed, Y. Tenenbaum-Rakover, and S. Refetoff, "A familial thyrotropin (TSH) receptor mutation provides in vivo evidence that the inositol phosphates $/ \mathrm{Ca}^{2+}$ cascade mediates TSH action on thyroid hormone synthesis," The Journal of Clinical Endocrinology \& Metabolism, vol. 92, no. 7, pp. 2816-2820, 2007.

[4] Y. Maeda and T. Kinoshita, "Structural remodeling, trafficking and functions of glycosylphosphatidylinositol-anchored proteins," Progress in Lipid Research, vol. 50, no. 4, pp. 411-424, 2011.

[5] M. J. Berridge, "Inositol trisphosphate and calcium oscillations," Advances in Second Messenger and Phosphoprotein Research, vol. 26, pp. 211-223, 1992.

[6] C. P. Downes and C. H. Macphee, "Myo-inositol metabolites as cellular signals," European Journal of Biochemistry, vol. 193, no. 1, pp. 1-18, 1990.

[7] X. Shen, H. Xiao, R. Ranallo, W.-H. Wu, and C. Wu, "Modulation of ATP-dependent chromatin-remodeling complexes by inositol polyphosphates,' Science, vol. 299, no. 5603, pp. 112-114, 2003.

[8] K. Strange, R. Morrison, C. W. Heilig, S. DiPietro, and S. R. Gullans, "Upregulation of inositol transport mediates inositol accumulation in hyperosmolar brain cells," American Journal of Physiology-Cell Physiology, vol. 260, no. 4, part 1, pp. C784C790, 1991.

[9] L. C. Huang, M. C. Fonteles, D. B. Houston, C. Zhang, and J. Larner, "Chiroinositol deficiency and insulin resistance. III. Acute glycogenic and hypoglycemic effects of two inositol phosphoglycan insulin mediators in normal and streptozotocindiabetic rats in vivo," Endocrinology, vol. 132, no. 2, pp. 652-657, 1993.

[10] F. Facchinetti, M. Bizzarri, S. Benvenga et al., "Results from the International Consensus Conference on Myo-inositol and Dchiro-inositol in Obstetrics and Gynecology: the link between metabolic syndrome and PCOS," European Journal of Obstetrics Gynecology and Reproductive Biology, vol. 195, pp. 72-76, 2015.

[11] A. Bevilacqua, G. Carlomagno, S. Gerli et al., "Results from the international consensus conference on myo-inositol and Dchiro-inositol in obstetrics and gynecology-assisted reproduction technology," Gynecological Endocrinology, vol. 31, no. 6, pp. 441-446, 2015.

[12] A. Bevilacqua and M. Bizzarri, "Physiological role and clinical utility of inositols in polycystic ovary syndrome," Best Practice \& Research. Clinical Obstetrics \& Gynaecology, 2016.

[13] W. A. March, V. M. Moore, K. J. Willson, D. I. W. Phillips, R. J. Norman, and M. J. Davies, "The prevalence of polycystic ovary syndrome in a community sample assessed under contrasting diagnostic criteria," Human Reproduction, vol. 25, no. 2, pp. 544-551, 2010.

[14] A. Dunaif, K. R. Segal, W. Futterweit, and A. Dobrjansky, "Profound peripheral insulin resistance, independent of obesity, in polycystic ovary syndrome," Diabetes, vol. 38, no. 9, pp. 11651174, 1989.

[15] F. Facchinetti, G. Dante, and I. Neri, "The ratio of MI to DCI and its impact in the treatment of polycystic ovary syndrome: experimental and literature evidences," in Frontiers in Gynecological Endocrinology, A. R. Genazzani and B. C. Tarlatzis, Eds., ISGE Series, pp. 103-109, 2016.

[16] S. Colazingari, M. Treglia, R. Najjar, and A. Bevilacqua, "The combined therapy myo-inositol plus D-chiro-inositol, rather than D-chiro-inositol, is able to improve IVF outcomes: results from a randomized controlled trial," Archives of Gynecology and Obstetrics, vol. 288, no. 6, pp. 1405-1411, 2013.

[17] P. Beemster, P. Groenen, and R. Steegers-Theunissen, "Involvement of inositol in reproduction," Nutrition Reviews, vol. 60, no. 3, pp. 80-87, 2002.

[18] L. M. Lewin and R. Beer, "Prostatic secretion as the source of myo inositol in human seminal fluid," Fertility and Sterility, vol. 24, no. 9, pp. 666-670, 1973.

[19] B. T. Hinton, R. W. White, and B. P. Setchell, "Concentrations of myo-inositol in the luminal fluid of the mammalian testis and epididymis," Journal of Reproduction and Fertility, vol. 58, no. 2, pp. 395-399, 1980.

[20] R. A. Condorelli, S. La Vignera, S. Bellanca, E. Vicari, and A. E. Calogero, "Myoinositol: does it improve sperm mitochondrial function and sperm motility?” Urology, vol. 79, no. 6, pp. 12901295, 2012.

[21] R. A. Condorelli, S. La Vignera, F. Di Bari, V. Unfer, and A. E. Calogero, "Effects of myoinositol on sperm mitochondrial function in-vitro," European Review for Medical and Pharmacological Sciences, vol. 15, no. 2, pp. 129-134, 2011.

[22] L. M. Lewin, Y. Yannai, S. Melmed, and M. Weiss, "Myo-inositol in the reproductive tract of the female rat," International Journal of Biochemistry, vol. 14, no. 2, pp. 147-150, 1982.

[23] T. T. Y. Chiu, M. S. Rogers, E. L. K. Law, C. M. Briton-Jones, L. P. Cheung, and C. J. Haines, "Follicular fluid and serum concentrations of myo-inositol in patients undergoing IVF: relationship with oocyte quality," Human Reproduction, vol. 17, no. 6, pp. 1591-1596, 2002.

[24] I. P. C. Krapels, I. A. L. M. Van Rooij, R. A. Wevers et al., "Myo-inositol, glucose and zinc status as risk factors for nonsyndromic cleft lip with or without cleft palate in offspring: a case-control study," BJOG: An International Journal of Obstetrics and Gynaecology, vol. 111, no. 7, pp. 661-668, 2004.

[25] D. Heimark, J. McAllister, and J. Larner, "Decreased myoinositol to chiro-inositol (M/C) ratios and increased $\mathrm{M} / \mathrm{C}$ epimerase activity in PCOS theca cells demonstrate increased insulin sensitivity compared to controls," Endocrine Journal, vol. 61, no. 2, pp. 111-117, 2014

[26] M. Bizzarri, A. Cucina, S. Dinicola et al., "Does myo-inositol effect on PCOS follicles involve cytoskeleton regulation?" Medical Hypotheses, vol. 91, pp. 1-5, 2016.

[27] H. M. Kwon, A. Yamauchi, S. Uchida et al., "Cloning of the cDNa for a $\mathrm{Na}^{+}$/myo-inositol cotransporter, a hypertonicity stress protein," The Journal of Biological Chemistry, vol. 267, no. 9, pp. 6297-6301, 1992.

[28] B. D. Higgins and M. T. Kane, "Inositol transport in mouse oocytes and preimplantation embryos: effects of mouse strain, embryo stage, sodium and the hexose transport inhibitor, phloridzin," Reproduction, vol. 125, no. 1, pp. 111-118, 2003.

[29] M. M. Zacchè, L. Caputo, S. Filippis, G. Zacchè, M. Dindelli, and A. Ferrari, "Efficacy of myo-inositol in the treatment of 
cutaneous disorders in young women with polycystic ovary syndrome," Gynecological Endocrinology, vol. 25, no. 8, pp. 508513, 2009.

[30] M. Matsuda, K. Tsutsumi, T. Kanematsu et al., "Involvement of phospholipase C-related inactive protein in the mouse reproductive system through the regulation of gonadotropin levels," Biology of Reproduction, vol. 81, no. 4, pp. 681-689, 2009.

[31] P. T. Goud, A. P. Goud, P. Van Oostveldt, and M. Dhont, "Presence and dynamic redistribution of type I inositol 1,4,5trisphosphate receptors in human oocytes and embryos during in-vitro maturation, fertilization and early cleavage divisions," Molecular Human Reproduction, vol. 5, no. 5, pp. 441-451, 1999.

[32] K. M. Lowther, V. N. Weitzman, D. Maier, and L. M. Mehlmann, "Maturation, fertilization, and the structure and function of the endoplasmic reticulum in cryopreserved mouse oocytes," Biology of Reproduction, vol. 81, no. 1, pp. 147-154, 2009.

[33] T. T. Y. Chiu, M. S. Rogers, C. Briton-Jones, and C. Haines, "Effects of myo-inositol on the in-vitro maturation and subsequent development of mouse oocytes," Human Reproduction, vol. 18, no. 2, pp. 408-416, 2003.

[34] M. T. Kane, M. Norris, and R. A. P. Harrison, "Uptake and incorporation of inositol by preimplantation mouse embryos," Journal of Reproduction and Fertility, vol. 96, no. 2, pp. 617-625, 1992.

[35] S. M. Warner, F. V. Conlon, and M. T. Kane, "Inositol transport in preimplantation rabbit embryos: effects of embryo stage, sodium, osmolality and metabolic inhibitors," Reproduction, vol. 125, no. 4, pp. 479-493, 2003, Erratum in: Reproduction, vol. 129, no. 1, pp. 128, 2005.

[36] P. Holm, P. J. Booth, M. H. Schmidt, T. Greve, and H. Callesen, "High bovine blastocyst development in a static in vitro production system using SOFaa medium supplemented with sodium citrate and myo-inositol with or without serum-proteins," Theriogenology, vol. 52, no. 4, pp. 683-700, 1999.

[37] L. Keskintepe, C. A. Burnley, and B. G. Brackett, "Production of viable bovine blastocysts in defined in vitro conditions," Biology of Reproduction, vol. 52, no. 6, pp. 1410-1417, 1995.

[38] S. Colazingari, M. T. Fiorenza, G. Carlomagno, R. Najjar, and A. Bevilacqua, "Improvement of mouse embryo quality by myoinositol supplementation of IVF media," Journal of Assisted Reproduction and Genetics, vol. 31, no. 4, pp. 463-469, 2014.

[39] D. K. Gardner, L. Reed, D. Linck, C. Sheehan, and M. Lane, "Quality control in human in vitro fertilization," Seminars in Reproductive Medicine, vol. 23, no. 4, pp. 319-324, 2005.

[40] J. P. E. M. Punt-van der Zalm, J. C. M. Hendriks, J. R. Westphal, J. A. M. Kremer, S. Teerenstra, and A. M. M. Wetzels, “Toxicity testing of human assisted reproduction devices using the mouse embryo assay," Reproductive BioMedicine Online, vol. 18, no. 4, pp. 529-535, 2009.

[41] T. Kaneko and R. Ohno, "Improvement in the development of oocytes from C57BL/6 mice after sperm injection," Journal of the American Association for Laboratory Animal Science, vol. 50, no. 1, pp. 33-36, 2011.

[42] L. M. Mehlmann and D. Kline, "Regulation of intracellular calcium in the mouse egg: calcium release in response to sperm or inositol trisphosphate is enhanced after meiotic maturation," Biology of Reproduction, vol. 51, no. 6, pp. 1088-1098, 1994.

[43] A. Pesty, N. Avazeri, and B. Lefèvre, "Nuclear calcium release by InsP3-receptor channels plays a role in meiosis reinitiation in the mouse oocyte," Cell Calcium, vol. 24, no. 4, pp. 239-251, 1998.
[44] S. Persad, S. Attwell, V. Gray et al., "Regulation of protein kinase B/Akt-serine 473 phosphorylation by integrin-linked kinase: critical roles for kinase activity and amino acids arginine 211 and serine 343," The Journal of Biological Chemistry, vol. 276, no. 29, pp. 27462-27469, 2001.

[45] M. T. Fiorenza, S. Torcia, S. Canterini et al., "TCL1 promotes blastomere proliferation through nuclear transfer, but not direct phosphorylation, of AKT/PKB in early mouse embryos," Cell Death and Differentiation, vol. 15, no. 2, pp. 420-422, 2008.

[46] M. L. Croze, R. E. Vella, N. J. Pillon et al., "Chronic treatment with myo-inositol reduces white adipose tissue accretion and improves insulin sensitivity in female mice," Journal of Nutritional Biochemistry, vol. 24, no. 2, pp. 457-466, 2013.

[47] A. Klippel, W. M. Kavanaugh, D. Pot, and L. T. Williams, "A specific product of phosphatidylinositol 3-kinase directly activates the protein kinase Akt through its pleckstrin homology domain," Molecular and Cellular Biology, vol. 17, no. 1, pp. 338344, 1997.

[48] J. G. Quirk Jr. and J. E. Bleasdale, "Myo-inositol homeostasis in the human fetus," Obstetrics and Gynecology, vol. 62, no. 1, pp. 41-44, 1983.

[49] R. D’Anna, V. Di Benedetto, P. Rizzo et al., "Myo-inositol may prevent gestational diabetes in PCOS women," Gynecological Endocrinology, vol. 28, no. 6, pp. 440-442, 2012.

[50] R. D’Anna, A. Scilipoti, D. Giordano et al., "Myo-inositol supplementation and onset of gestational diabetes mellitus in pregnant women with a family history of type 2 diabetes: a prospective, randomized, placebo-controlled study," Diabetes Care, vol. 36, no. 4, pp. 854-857, 2013.

[51] N. D. E. Greene and A. J. Copp, "Inositol prevents folateresistant neural tube defects in the mouse," Nature Medicine, vol. 3, no. 1, pp. 60-66, 1997.

[52] Z. Dai, S. K. Chung, D. Miao, K. S. Lau, A. W. H. Chan, and A. W. C. Kung, "Sodium/myo-inositol cotransporter 1 and myo-inositol are essential for osteogenesis and bone formation," Journal of Bone and Mineral Research, vol. 26, no. 3, pp. 582-590, 2011.

[53] J. F. L. Chau, M. K. Lee, J. W. S. Law, S. K. Chung, and S. S. M. Chung, "Sodium/myo-inositol cotransporter-1 is essential for the development and function of the peripheral nerves," The FASEB Journal, vol. 19, no. 13, pp. 1887-1889, 2005.

[54] E. A. Reece, M. Khandelwal, Y.-K. Wu, and M. Borenstein, "Dietary intake of myo-inositol and neural tube defects in offspring of diabetic rats," American Journal of Obstetrics and Gynecology, vol. 176, no. 3, pp. 536-539, 1997.

[55] A. Nagy, M. Gertsenstein, K. Vintersten, and R. Behringer, Manipulating the Mouse Embryo-A Laboratory Manual, Cold Spring Harbor Laboratory Press, Cold Spring Harbor, NY, USA, 3rd edition, 2003.

[56] J. Downward, "Mechanisms and consequences of activation of protein kinase B/Akt," Current Opinion in Cell Biology, vol. 10, no. 2, pp. 262-267, 1998.

[57] A. Khwaja, P. Rodriguez-Viciana, S. Wennström, P. H. Warne, and J. Downward, "Matrix adhesion and Ras transformation both activate a phosphoinositide 3-OH kinase and protein kinase B/Akt cellular survival pathway," EMBO Journal, vol. 16, no. 10, pp. 2783-2793, 1997.

[58] A. Toker and A. C. Newton, "Akt/protein kinase B is regulated by autophosphorylation at the hypothetical PDK-2 site," The Journal of Biological Chemistry, vol. 275, no. 12, pp. 8271-8274, 2000 . 
[59] A. Toker and A. C. Newton, "Cellular signaling: pivoting around PDK-1," Cell, vol. 103, no. 2, pp. 185-188, 2000.

[60] R. M. Biondi, P. C. F. Cheung, A. Casamayor, M. Deak, R. A. Currie, and D. R. Alessi, "Identification of a pocket in the PDK1 kinase domain that interacts with PIF and the C-terminal residues of PKA," The EMBO Journal, vol. 19, no. 5, pp. 979-988, 2000.

[61] S. Dinicola, G. Fabrizi, M. G. Masiello et al., "Inositol induces mesenchymal-epithelial reversion in breast cancer cells through cytoskeleton rearrangement," Experimental Cell Research, vol. 345, no. 1, pp. 37-50, 2016.

[62] M. Azadbakht, M. R. Valojerdi, and S. J. Mowla, "Development of mouse embryos co-cultured with polarized or non-polarized uterine epithelial cells using sequential culture media," Animal Reproduction Science, vol. 100, no. 1-2, pp. 141-157, 2007.

[63] R. Fernández-Gonzalez, P. Moreira, A. Bilbao et al., "Longterm effect of in vitro culture of mouse embryos with serum on mRNA expression of imprinting genes, development, and behavior," Proceedings of the National Academy of Sciences of the United States of America, vol. 101, no. 16, pp. 5880-5885, 2004. 


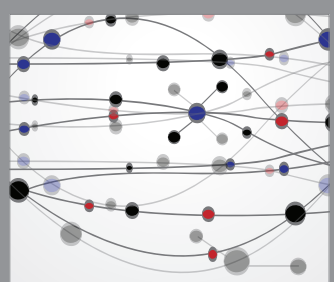

The Scientific World Journal
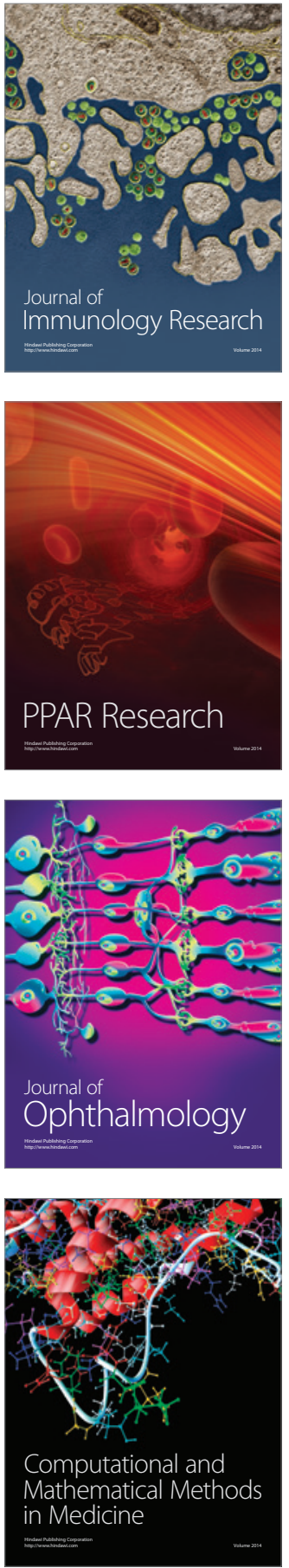



Gastroenterology Research and Practice

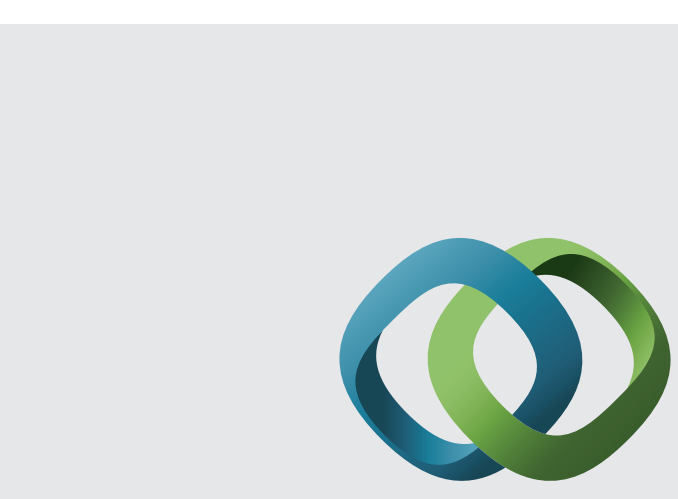

\section{Hindawi}

Submit your manuscripts at

http://www.hindawi.com

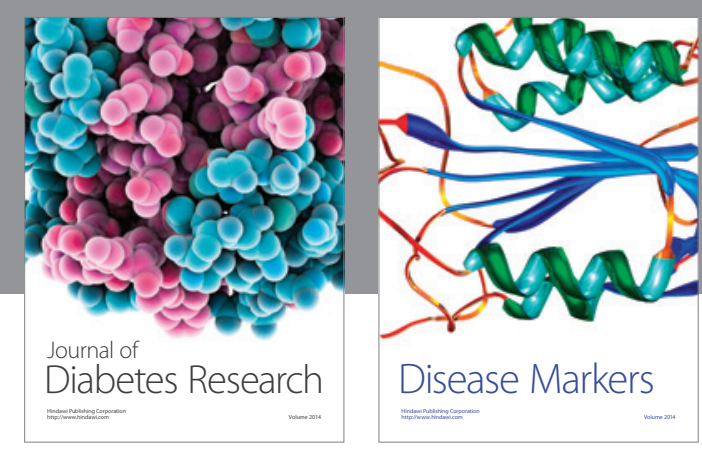

Disease Markers
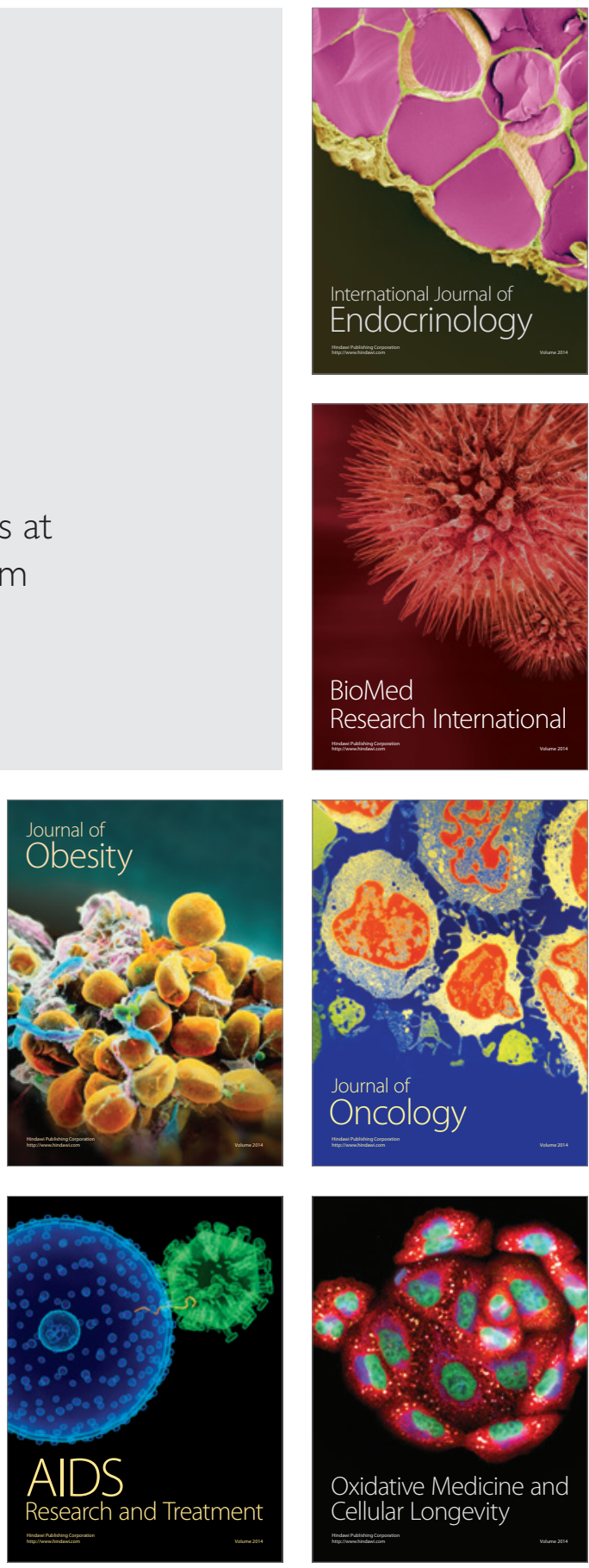